\title{
Expert Systems With Applications
}

\section{Integrated technique of segmentation and classification methods with connected components analysis for road extraction from orthophoto images

\author{
--Manuscript Draft--
}

\begin{tabular}{|c|c|}
\hline Manuscript Number: & ESWA-D-19-05213R2 \\
\hline Keywords: & $\begin{array}{l}\text { road extraction; image segmentation; image classification; connected components } \\
\text { analysis; Remote Sensing }\end{array}$ \\
\hline Corresponding Author: & $\begin{array}{l}\text { Biswajeet Pradhan, PhD } \\
\text { University of Technology Sydney } \\
\text { Sydney, AUSTRALIA }\end{array}$ \\
\hline First Author: & Abolfazl Abdollahi, Ph.D., \\
\hline \multirow[t]{2}{*}{ Order of Authors: } & Abolfazl Abdollahi, Ph.D., \\
\hline & Biswajeet Pradhan, PhD \\
\hline Abstract: & $\begin{array}{l}\text { Road networks are one of the main urban features. Therefore, road parts extraction } \\
\text { from high-resolution remotely sensed imagery and updated road database are } \\
\text { beneficial for many GIS applications. However, owing to the presence of various types } \\
\text { of obstacles in the images, such as shadows, cars, and trees, with similar transparency } \\
\text { and spectral values as road class, achieving accurate road extraction using different } \\
\text { classification and segmentation methods is still difficult. This paper proposes an } \\
\text { integrated method combining segmentation and classification methods with connected } \\
\text { components analysis to extract road class from orthophoto images. The proposed } \\
\text { technique is threefold. First, multiresolution segmentation method was applied to } \\
\text { segment images. Then, the main classification methods, namely, decision trees (DT), } \\
\text { k-nearest neighbors (KNN), and support vector machines (SVM), were implemented } \\
\text { based on spectral, geometric, and textural information to classify the obtained results } \\
\text { into two classes: road and non-road. Three main accuracy evaluation measures, such } \\
\text { as recall, precision, and F1-score, were evaluated to determine the performance of the } \\
\text { proposed method, with respective average values of } 87.62 \%, 89.71 \% \text {, and } 88.61 \% \text {, } \\
\text { respectively, for DT; } 86.61 \%, 88.17 \% \text {, and } 87.30 \% \text {, respectively, for KNN; and } 89.83 \% \text {, } \\
89.52 \% \text {, and } 89.67 \% \text {, respectively, for SVM. Finally, connected components labelling } \\
\text { was used to extract road component parts, and morphological operation was employed } \\
\text { to delete non-road parts and noises and improve the performance. These results were } \\
\text { also compared with other prior works, which confirmed that the integrated method is an } \\
\text { effective road extraction technique. }\end{array}$ \\
\hline
\end{tabular}


(c) <2021 > . This manuscript version is made available under the CC-BY-NC-ND 4.0 license http://creativecommons.org/licenses/by-nc-nd/4.0/

The definitive publisher version is available online at https://doi.org/ $\underline{10.1016 / \text { i.eswa.2021.114908 }}$ 


\title{
Integrated technique of segmentation and classification methods with connected components analysis for road extraction from orthophoto images
}

\author{
Abolfazl Abdollahi' ${ }^{1}$ and Biswajeet Pradhan ${ }^{1,2,3 *}$ \\ ${ }^{1}$ Centre for Advanced Modelling and Geospatial Information Systems (CAMGIS), $\underline{\text { School of }}$ \\ Information, Systems and Modeling, Faculty of Engineering and IT, University of \\ Technology Sydney, NSW 2007, Australia \\ Email: abolfazl.abdollahi@student.uts.edu.au \\ ${ }^{2}$ Department of Energy and Mineral Resources Engineering, Sejong University, Choongmu- \\ gwan, 209 Neungdong-ro, Gwangjingu, Seoul 05006, Korea \\ ${ }^{3}$ Center of Excellence for Climate Change Research, King Abdulaziz University, P. O. Box \\ 80234, Jeddah 21589, Saudi Arabia \\ * Correspondence: biswajeet24@ gmail.com or Biswajeet.Pradhan@uts.edu.au
}




\section{Abstract}

Road networks are one of the main urban features. Therefore, road parts extraction from highresolution remotely sensed imagery and updated road database are beneficial for many GIS applications. However, owing to the presence of various types of obstacles in the images, such as shadows, cars, and trees, with similar transparency and spectral values as road class, achieving accurate road extraction using different classification and segmentation methods is still difficult. This paper proposes an integrated method combining segmentation and classification methods with connected components analysis to extract road class from orthophoto images. The proposed technique is threefold. First, multiresolution segmentation method was applied to segment images. Then, the main classification methods, namely, decision trees (DT), k-nearest neighbors (KNN), and support vector machines (SVM), were implemented based on spectral, geometric, and textural information to classify the obtained recall, precision, and F1-score, were evaluated to determine the performance of the proposed method, with respective average values of $87.62 \%, 89.71 \%$, and $88.61 \%$, respectively, for DT; $86.61 \%, 88.17 \%$, and $87.30 \%$, respectively, for $\mathrm{KNN}$; and $89.83 \%, 89.52 \%$, and $89.67 \%$, respectively, for SVM. Finally, connected components labelling was used to extract road component parts, and morphological operation was employed to delete non-road parts and noises and improve the performance. These results were also compared with other prior works, which confirmed that the integrated method is an effective road extraction technique.

Keywords: road extraction; image segmentation; image classification; connected components analysis; remote sensing

\section{Introduction}

With the revolution of new generation remote sensing technologies, high-resolution remote sensing imagery has become frequently accessible recently. Image processing and interpretation are necessary to analyze remote sensing images because a massive number of images are captured by these sensors (Grinias, Panagiotakis, \& Tziritas, 2016). Among the remote sensing fields, road network extraction from remote sensing images with high spatial resolution is a considerable subject that received ample attention from researchers in recent 
years (Rezaee \& Zhang, 2017). Compared to low and medium spatial resolution images, road parts are displayed in the high-resolution remotely sensed imagery with comprehensive spatial information. Regular updates of road network database are required because the urban environment is rapidly shifting (Abdollahi, et al., 2020). Road lengths are lengthy and generally longer than those of street blocks and buildings, while road width is usually a few pixels in remote sensing imagery (Sujatha \& Selvathi, 2015). Therefore, precise road network extraction from very high-resolution remotely sensed images is necessary for different kinds of urban applications, such as updating maps in geographic information system (Abdollahi, Pradhan, \& Shukla, 2019), road navigation (Li, Jin, Fei, \& Ma, 2014), land cover analysis (Zhang, Chen, Zhuo, Geng, \& Wang, 2018), and transportation and traffic management (Liu, Wu, Wang, \& Liu, 2015). However, owing to existing obstructions and noise in these images, such as contextual structures (shadows, vehicles, vegetation, and trees) and road-like features (such as car parking and railways), which have similar spectral and spatial characteristics and produce heterogeneous areas causing the incorrect segmentation of road parts, extracting road parts from remotely sensed imagery becomes a challenging task (Li, et al., 2019). Manual road extraction from high-resolution remote sensing images is inefficient and very time- and costconsuming, thus failing to satisfy real-time processing requests; semi-automatic and automatic approaches are preferred (Courtrai \& Lefèvre, 2016). Several machine learning methods, such as support vector machine (SVM) (Guo, et al., 2016), random forest (RF) (Rodriguez-Galiano, Ghimire, Rogan, Chica-Olmo, \& Rigol-Sanchez, 2012), maximum likelihood (Ahmad \& Quegan, 2012), and neural networks (Ratle, Camps-Valls, \& Weston, 2010), which are pixelbased traditional classification approaches, only rely on the spectral information of the images. Pixel-wise classifiers have one limitation, that is, they are subject to the color phenomena; this means that these methods classify the images based on color reflectance, which leads to loss of portions with similar color and background (Fauvel, Chanussot, \& Benediktsson, 2012). Therefore, integrating segmentation and classification methods that provide high accuracy by utilizing spectral information along with the spatial and texture information is gaining considerable attention in the remote sensing field.

\section{Related works}

Road network extraction from high-resolution remote sensing imagery can be divided into automatic and semi-automatic approaches (Khesali, Zoej, Mokhtarzade, \& Dehghani, 2016). User input as prior information is needed for semi-automatic techniques (Chaudhuri, Kushwaha, \& Samal, 2012), whereas automatic techniques do not need any prior information 
(Mnih \& Hinton, 2010). Miao, Shi, Gamba, and Li (2015) applied an approach derived from semi-automatic approaches to extract road centerline from very high resolution (VHR) imagery. They first used the geodesic technique to exploit the primary road sections and produce the probability map. Then, they utilized thresholding operation to classify the image in non-road and road parts. The obtained results showed that the suggested technique can accurately and rapidly detect centerline of roads from VHR images.

In the work of (Alshehhi \& Marpu, 2017), hierarchal graph-based segmentation was introduced for road part extraction from high-resolution remote sensing imagery. This technique includes three main steps: (1) pre-processing, which is based on morphological and Gabor filtering to extract features and intensifies the contradiction between non-road and road sections; (2) graph-based segmentation, which is based on hierarchical joining and the dividing of the image segments using shape and color characteristics; and (3) post-processing, which is applied to remove small artefact features in the extracted road sections and improve accuracy. The outcomes proved that this technique is superior for road parts extraction from highresolution remote sensing imagery in an urban area.

In a recent paper, Shen, Ai, and Yang (2019) proposed a novel approach called superpixel centerline extraction to extract dual-line roads from remotely sensed images. First, they used simple linear iterative clustering to segment dual-line roads. Next, the superpixels situated at road intersections were merged to generate connection points from their skeleton. Finally, they connected the midpoints and center points of edges of every superpixel to generate road centerlines. The extracted road centerline was tested using an old vector data at a scale of 1:50,000. They found that the proposed method can eliminate noises and yield an excellent road extraction result from simple and complex road intersections.

Gao, et al. (2018) introduced a multiple feature pyramid network for extracting a road class from remotely sensed images. They also presented the weighted balance loss function to settle the class unbalance difficulty produced by the sparseness of road sections. They found that compared with cross-entropy loss function, training time can be dramatically decreased by the weighted loss function. Two datasets were used to test their proposed method, and the results confirmed that the method can obtain high accuracy for road class extraction. A semi-automatic approach is presented by (Khesali, et al., 2016) for road class extraction from IKONOS and TerraSAR-X imagery. They applied an integrated knowledge-based and neural network approach using spectral and texture information for road extraction. The results proved that the proposed approach is effective for extracting road portions. Kamangir, Momeni, and Satari 
(2017) performed maximum likelihood method, morphological operations, and random sample consensus approach for image classification, segmented image rectification, and road class extraction, respectively. The obtained completeness factor was $85 \%$, indicating the effectiveness of the suggested approach for road extraction. Da-Ming, Xiang, and Chun-Li (2011) suggested a method for road network extraction based on Markov random field (MRF), SVM, and fuzzy c-mean (FCM). They integrated the latter two models to extract road section and then compared the outcome with that of the MRF method. They found that the fusion method of SVM and FCM is more effective than the MRF method for extracting road class from remotely sensed imagery.

A combined approach of SVM and level set (LS) is applied by (Abolfazl Abdollahi, Bakhtiari, \& Nejad, 2018) for extracting road regions from google earth imagery. They achieved some common measures, such as completeness and correctness, and realized that the integrated technique is efficient in extracting road class. A new approach based on graph theoretical technique for road network extraction from high-resolution remote sensing imagery was introduced by (Unsalan \& Sirmacek, 2012). Various kinds of images, such as QuickBird, IKONOS, and GeoEye, were used to designate the deficiencies and robustness of the recommended system. The empirical results demonstrated that the suggested technique can efficiently extract road parts. Revathi and Sharmila (2013) applied pre-processing approach to increase the quality of images by removing noises first. They then implemented SVM and mean shift approach to extract road portions from IKONOS images. They obtained completeness and correctness metrics, which show that the proposed model achieved good results in road part extraction. Singh and Garg (2013) extracted road parts using a combination of morphological operators and adaptive global thresholding. The thresholding method was applied to segment roads, whereas the morphological operators were utilized to fill the gaps and improve accuracy. They discovered that the suggested model could achieve acceptable results for road extraction based on the obtained performance measures (e.g., correctness and completeness).

A road centerline extraction approach was introduced by Sujatha \& Selvathi (2015) for road class extraction from high-resolution remote sensing imagery. They segmented the images and then used connected components operators to extract united road segments. They applied morphological operations to remove pixels of non-road sections. The outcome verified the robustness of the introduced method in road part extraction. Moreover, various shape characteristics with spectral features, such as compound feature set (Valero, Chanussot, Benediktsson, Talbot, \& Waske, 2010), image moments (Das, Mirnalinee, \& Varghese, 2011), 
morphological operations (Shi, Miao, Wang, \& Zhang, 2014), and linear feature index (Miao, Shi, Zhang, \& Wang, 2012), can be used to improve road class segmentation and classification. Shi, et al. (2014) performed a spectral-spatial classification method and shape features to extract road object from IKONOS and Ziyuan-3 satellite images. They first used opening and closing morphological operations to classify images into non-road and road sections. Local Geary's $C$ technique was then applied to obtain the homogeneity of local gray values. Finally, shape features, such as length and area, were used to improve the road part. The results depicted the effectiveness of the suggested approach in extracting road parts from high-resolution remotely sensed image. However, the method is unsuitable for extracting road class from lowresolution images with a spatial resolution below six meters.

Some eminent shape features were used by Zhang, et al., (2018) for road class extraction from remote sensing imagery. They first extracted road edge using singular value decomposition method and then constructed road sections using k-mean clustering approach. Next, a combination of eminent shape features and total variation-based image contraction approach was used to obtain road networks. Morphological operators were used to remove noises and extract non-road parts to improve accuracy. Completeness and correctness assessment measures were achieved and proved that the suggested technique is remarkable in detecting and extracting road class from remotely sensed imagery. Pixel-wise classification techniques rely on color and classify images based on feature color reflectance. Therefore, the main problem of these techniques is color sensitivity, which has motivated the authors of this paper to use other characteristics, such as spatial and texture features, to classify images and extract road class. Merging spectral, spatial, and textural information generally demonstrates better outcomes compared when only spectral information is used. Therefore, this paper aims to integrate segmentation and classification methods with connected components analysis using spectral values (mean and standard deviation), geometric information (area, length, and number of pixels), and textural features (entropy, contrast, homogeneity, and mean) to categorize orthophoto images into road and non-road class and extract the road parts. In addition, the utilization of additional shape saliency features can tackle the color sensitivity and improve the performance of road extraction methods.

Object-based image analysis (OBIA) usually has more benefits compared with that of traditional pixel-based classification approaches. For example, OBIA techniques consider not only spectral values but also textural and spatial features in classifying images, while pixelbased techniques depend only on a single pixel or its neighborhood information (Maboudi, 
Amini, Hahn, \& Saati, 2017). The performance of pixel-based classification techniques is generally lower than that of the OBIA when dealing with road extraction and VHR remotely sensed image classification (Blaschke, 2010). Therefore, the main contribution of this work is to offers an integrated model of segmentation and classification methods with connected components labeling for road extraction from orthophoto images. The following steps are conducted to achieve this goal. First, the segmentation technique is used to split the image into some segments, and then the results are processed using the classification methods to categorize the images into non-road and road sections. Then, the connected components labeling is applied to the final binary images to assert its pixels into components based on pixel connectivity to extract road parts and delete some components belonging to non-road sections. Finally, morphological operations are performed to remove noises, fill the gaps, and improve the performance. The training time for the proposed classification methods was relatively short, while providing satisfactory results for both quantitative and qualitative parts. Moreover, these methods were incorporated with the connected components labeling and morphological operation for road extraction from orthophoto images, which has not been performed in previous studies. The rest of this paper is organized as follows. The basic principle of the suggested approach is illustrated in Section 3. Section 4 explains the empirical outcomes. Section 5 and 6 report the discussion and conclusion parts, respectively.

\section{Materials and methodology}

\subsection{Proposed model}

An effective model for road section extraction from VHR remotely sensed images is presented in this work. This model has the following three steps. First, multiresolution segmentation was performed to divide the images into segments based on their spectral values. A total of 567 segments were selected as labeling data for training classification methods based on the segmented images randomly. Then, three main classification approaches, namely, SVM, decision tree (DT), and k-nearest neighbors $(\mathrm{KNN})$, were applied to the segmented image and trained based on sampling data to classify the image into two principal classes: road and non-road class. Finally, connected components analysis and morphological operations were performed to group the pixels together in terms of similar connected components and delete holes and noises to improve the accuracy of the proposed road extraction method. Figure 1 illustrates the flowchart 

images.

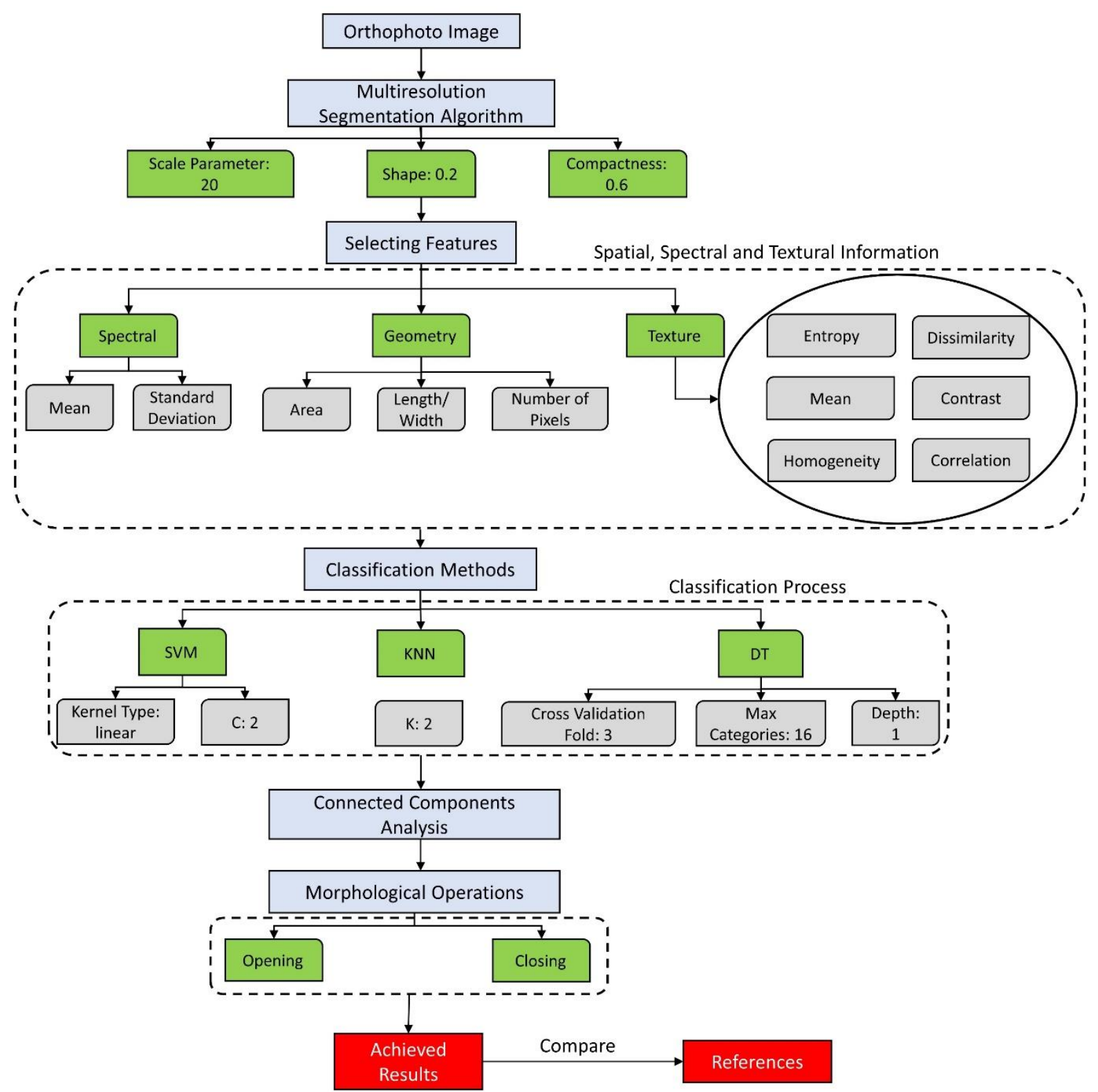

Fig. 1. Flowchart of the proposed road extraction method

\subsection{Segmentation process}

Image segmentation is a crucial step because it will produce the primary entities for the subsequent processes. The quality of image segmentation has a notable impact on the succeeding operations, making it a crucial yet challenging aspect of OBIA (Grote, Heipke, \& Rottensteiner, 2012). The algorithms for image segmentation can be divided into four main categories: edgebased, pixel-based, region-based, and mixture methods. The multiresolution segmentation technique is applied in this study for image segmentation (Saba, Valadan Zoej, \& Mokhtarzade, 
2016). The scale, shape, and compactness parameters for the proposed segmentation method were set to 20,0.2, and 0.6, respectively, to obtain high accuracy in the classification process. The proposed segmentation method is a region-based method, which reduces the nonhomogeneous segments using spectral and shape characteristics (Wang \& $\mathrm{Li}$, 2014). In this method, each pixel of the image is considered as an object. Then, using a fusion factor, objects were joined together to make a large one during a repetitive process. Equation 1 shows the fusion factor, which demonstrates the cost of fitting (Saba, et al., 2016).

$f=W_{\text {color }} h_{\text {color }}+W_{\text {shape }} h_{\text {shape }}$

where $\mathrm{h}_{\text {shape }}$ is the difference in the shape dissimilarity, $\mathrm{h}_{\text {color }}$ is the difference in the spectral dissimilarity, $\mathrm{W}_{\text {shape }}$ is the weight of shape dissimilarity, and $\mathrm{W}_{\text {color }}$ is the weight of spectral heterogeneity. Furthermore, $\mathrm{W}_{\text {color }}+\mathrm{W}_{\text {shape }}=1$. Equation 2 defines the difference between two objects on the basis of spectral heterogeneity in a multispectral image with B band.

$$
h_{\text {color }}=\sum_{b=1}^{B} W_{b}\left\{n_{m} \sigma_{b, m}-\left(n_{1} \sigma_{b, 1}+n_{2} \sigma_{b, 2}\right)\right\}
$$

where $\mathrm{n}$ is the number of pixels in every object; $\sigma$ is the standard deviation of spectral values; indexes 1,2 , and $\mathrm{m}$ represent the first, second, and the combined object, respectively; and $\mathrm{W}_{\mathrm{b}}$ is the band weight. Smoothness and compactness dissimilarity represent the difference between the shape heterogeneity of two objects (Maboudi, et al., 2017). The difference in shape dissimilarity is expressed by Equation 3. $\mathrm{W}_{\text {comp }}$ and $\mathrm{W}_{\text {smooth }}$ are the compactness and smoothness dissimilarities, respectively.

$$
\begin{aligned}
h_{\text {shape }}= & W_{\text {smooth }}\left\{n_{m} \frac{\ell_{m}}{p_{m}}-\left(n_{1} \frac{\ell_{1}}{p_{1}}+n_{2} \frac{\ell_{2}}{p_{2}}\right)\right\} \\
& +W_{\text {comp }}\left\{\ell_{m} \sqrt{n_{m}}-\left(\ell_{1} \sqrt{n_{1}}+\ell_{2} \sqrt{n_{2}}\right)\right\}
\end{aligned}
$$

where $\mathrm{p}$ shows the minimum bounding box perimeter of the object, and $\ell$ represents the genuine length of the object. $\mathrm{W}_{\text {smooth }}+\mathrm{W}_{\text {comp }}=1$.

\subsection{Selecting features}

In this paper, OBIA, which considers not only spectral information but also spatial and textural features, was applied to deal with color sensitivity and enhance the efficiency of the suggested road extraction approach. Pixels in the image are first grouped into objects on the basis of either spectral correlation or an outer parameter, such as ownership, soil, or geological unit in the OBIA (Blaschke, 2010). The parameter values, such as standard deviation and mean, were considered 
for each band in the image for the spectral values. The different shapes and elongation of road objects facilitated the easy identification of the proposed method. Geometric features (e.g., length/width, area, and number of pixels) were also considered to ease the classification process. Finally, for the textural values, contrast, entropy, dissimilarity, homogeneity, and correlation values were considered. These features are generally applied to alleviate the classification process and improve the efficiency of road extraction approaches. These features are fed into the classifiers as a training part to accurately classify the image into the road and non-road sections.

\subsection{Classification process}

After image segmentation, classifiers, such as SVM, KNN, and DT, were selected to categorize the orthophoto images into two principal classes: road and non-road. This section presents individual discussions of the above classifiers.

\subsubsection{SVM classifier}

SVM, which is one of the supervised machine learning approaches, exhibited ample ability in image classification compared with that of the traditional techniques, such as neural networks (X. Huang, Lu, \& Zhang, 2014). The SVM classifier is a linear classification approach that creates a hyperplane to separate data. The process of separating data into classes is followed by identifying the best hyperplane and maximum margin (Abolfazl Abdollahi, et al., 2018). SVM transforms data according to the predesignated sections in a novel space, wherein data can be detached and classified linearly. Then, a linear equation that provides a maximum margin between two classes is formulated by finding a support line in multi-dimensional space using SVM (Sghaier \& Lepage, 2016). The practical application of the SVM method depends on the hypothetical maximum margin classifier. Given that hyperplane is a line separating the input variable space, a hyperplane in the SVM classifier detaches points from the input variable space based on their class (0 or 1$)$. All the input points can be completely split by this line into a twodimension space (Equation 4).

$$
B_{0}+\left(B_{1} \times X_{1}\right)+\left(B_{2} \times X_{2}\right)=0
$$

where $\mathrm{X}_{1}$ and $\mathrm{X}_{2}$ are the input variable, $\mathrm{B}_{0}$ is set up by the learning algorithm, and $\mathrm{B}_{1}$ and $\mathrm{B}_{2}$ specify the slope of the line. In this study, the kernel type for SVM is considered to be a linear kernel explaining the distance measure or similarity between new data and support vectors. The performance of the SVM method is shown in Figure 2. The dotted lines in the figure represent corresponding class support vectors, and the data are presented into two categories (red and blue). 
The long black line is the SVM. Each kind of support vector has a characteristic formula that describes the boundary of each group.

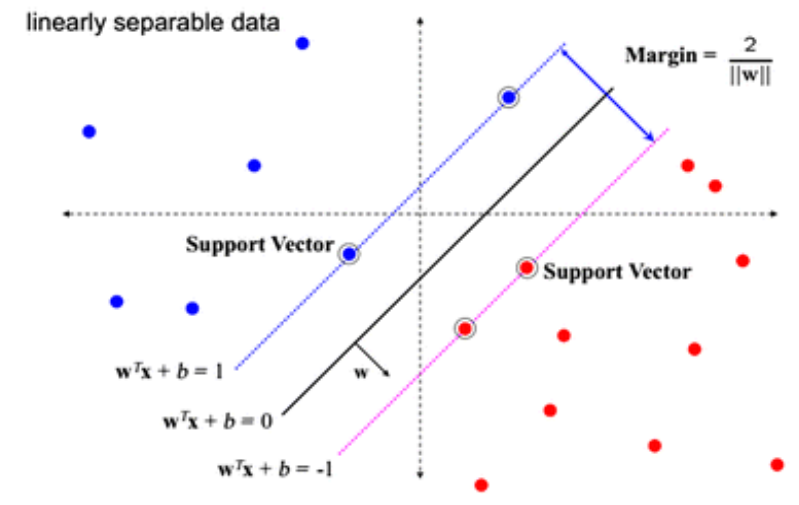

Fig. 2. SVM performance in categorizing data (Burges, 1998)

\subsubsection{KNN classifier}

One of the non-parametric techniques in machine learning methods is KNN, which has been utilized in statistical applications since the early 1970s (K. Huang, Li, Kang, \& Fang, 2016). The fundamental concept of $\mathrm{KNN}$ is the discovery of a collection of $\mathrm{k}$ samples in the calibration dataset nearest to uncertain samples based on distance functions. By evaluating the average of the response variables (e.g., attributes of KNN class), the class of uncertain samples is specified from these k samples (Akbulut, Sengur, Guo, \& Smarandache, 2017). Therefore, $\mathrm{k}$ is the key tuning parameter of $\mathrm{KNN}$ and plays a crucial role in ensuring the efficiency of $\mathrm{KNN}$ in image classification. The bootstrap process is used to identify the k parameter (Qian, Zhou, Yan, Li, \& Han, 2015). Different k values from 1 to 10 were inspected in this study to find the ideal $\mathrm{k}$ value from all the training datasets, which finally yielded 2 .

\subsubsection{DT algorithm}

Regarding the dispensation of data, the DT method can be executed without any previous statistical presumptions because it is a non-parametric classifier. The basic structure of the DT algorithm has three main parts, which include one root node, numerous interior nodes, and a collection of final nodes (Otukei \& Blaschke, 2010). The data are recessively broken down into a DT based on the assigned classification structure. Using a breaking test of the form $\mathrm{x}_{\mathrm{i}}>\mathrm{c}$ for univariate or $\sum_{i}^{n} a_{i} x_{i} \leq c$ for multivariate decision trees, a decision rule necessary at every node can be performed. Where $\mathrm{c}$ is the decision threshold, $\mathrm{a}$ is the linear coefficient vector, $\mathrm{n}$ is the chosen feature, and $\mathrm{x}_{\mathrm{i}}$ presents the evaluation vectors. Compared with traditional methods, such as the minimum-distance-to-means approach, the DT method has high precision. However, 
several variables, such as decision threshold, boosting, and pruning approaches, can affect the efficiency of DT in classification (Mishra, Singh, \& Yamaguchi, 2011). Some parameters, such as max categories, cross-validation fold, and depth, are set to 16,3 , and 1 , respectively, for the DT method to achieve optimal results.

\subsection{Connected component analysis and morphological operations}

After applying the classification methods and obtaining the results, connected components labeling was performed to extract road sections. Image pixels were grouped into components using connected components analysis on the basis of pixel connectivity, wherein all pixels in the connected component have the same pixel intensity values and are labeled with color or gray level based on each component (Vijayan \& Jyothy, 2016). The image can be partitioned into segments using these connected components. Morphological operators can be used to extract connected components. Analyzing connected components can be very useful for several applications, such as line detection and road extraction (Sujatha \& Selvathi, 2015).

The trivial operation was applied to extract connected component based on some criteria. Assume that $\mathrm{P}(\mathrm{i})$ is the connected component, $\mathrm{P}$ is the image, and $\mathrm{T}$ is the length of the main axis. The trivial opening can then be expressed as follows:

$R_{0}=\{P \mid$ Long axis of minimum ellipse enclosing $P(i) \geq T\}$

where $\mathrm{R}_{0}$ is the connected component. According to the $\mathrm{T}$, trivial operation is utilized for suitable connected components extraction. The entire region of connected components is preserved if that component satisfied condition $\mathrm{T}$ and is removed otherwise. After extracting the required connected components in terms of road section, common morphological operations, such as opening and erosion operations, were used to fill gaps, remove noises, delete non-road parts from the image, and improve the accuracy of the extracted road class using the proposed methods (Bakhtiari, Abdollahi, \& Rezaeian, 2017; Yadav \& Agrawal, 2018).

\subsection{Accuracy evaluation}

The road layer from orthophoto images was manually digitized using ArcMap software to compare it with the extracted road class and calculate the accuracy of the proposed road extraction technique. A confusion matrix containing road and non-road class pixels was used to assess the effectiveness of the proposed method in extracting road section. Some common metrics, such as recall (completeness) factor, F1-score, and precision (correctness) factors, were 
346 determined and presented in Equations (6), (7), and (8), respectively. The amount of road pixels extracted among all real road pixels is determined by the recall factor. A fusion of precision and recall is considered being the F1-score, while the precision factor determines the number of accurately extracted road pixels among all estimated pixels.

$350 \quad F 1=\frac{2 \times \text { Precision } \times \text { Recall }}{\text { Precision }+ \text { Recall }}$

$351 \quad$ Recall $=\frac{T P}{T P+F N}$

352 Precision $=\frac{T P}{T P+F P}$

\section{4. Results}

\subsection{Orthophotos and geometric correction}

Orthophoto images obtained from the state of Selangor in Peninsular Malaysia with spatial resolution of $7 \mathrm{~cm}$ are utilized in this paper (Figure 3). An Optech Airborne Laser Terrain Mapper 3100 instrument in an airborne laser scanning of light detection and ranging (LiDAR) system was used to collect orthophotos from the specific area on November 2, 2015. A LiDAR system basically includes a specific GPS (global positioning system) receptor, a scanner, and a laser. The most regularly utilized platforms for collecting LiDAR data over large regions are helicopters and airplanes. Laser scanning systems are classified as topographic and bathymetric. Topographic LiDAR maps the land based on a near-infrared laser, whereas bathymetric LiDAR measures seafloor and riverbed elevation and maps land based on water-penetrating green light (Ferraz, Mallet, \& Chehata, 2016). The flight height for data collection was $1510 \mathrm{~m}$ in a bright sky. The geometric calibration of the orthophoto images was performed to eliminate geometric error and designate single pixels in their appropriate planimetric $(\mathrm{x}, \mathrm{y})$ map positions (Aasen, Honkavaara, Lucieer, \& Zarco-Tejada, 2018). Subsequently, several well-distributed ground control points in the entire image were selected, and then the least square technique was performed to determine the coefficient. Finally, polynomial equations were formulated to determine the root mean shift error between the $\mathrm{X}, \mathrm{Y}$ of reference, and the adjusted coordinates. 


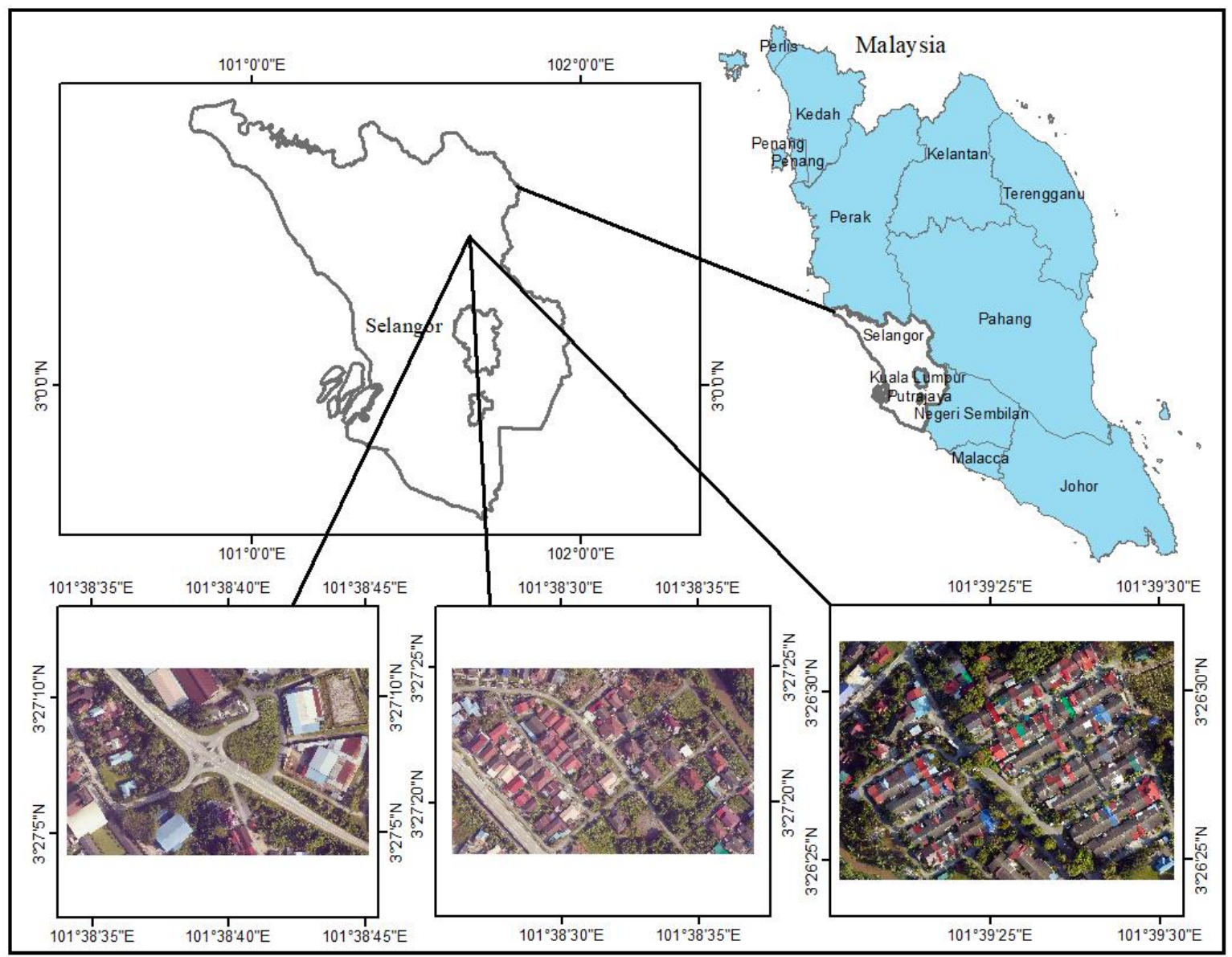

Fig. 3. Orthophoto images showing the location of the study area

\subsection{Experimental results}

In this study, a new method integrating segmentation and classification methods with connected components labeling was introduced to extract road class from different orthophoto images with different backgrounds. Three images from different areas, in which road section is covered by some other objects, such as vegetation, vehicles, and buildings, were considered to demonstrate the efficiency of the proposed road extraction method. Software, including MATLAB, eCognition Developer 64, and ArcMap, were used to apply the proposed method and calculate its efficiency in road extraction. We considered two sets of values for parameters such as scale, shape and compactness for the proposed segmentation approach to measure how the parameters of the method affect the detection accuracy. First, we set the values for the scale, shape and compactness parameters of the segmentation method to 50, 0.5 and 0.3 and then applied the classification methods, and the results are shown in Figure 4. Whereas Figure 5 shows the results of road detection by the methods after setting the values of scale, shape and compactness parameters to 20,0.2 and 0.6, respectively. Both figures are illustrated in five columns and three rows. The first and second columns depict the original RGB images and 
original ground truth maps, respectively. The third, fourth and fifth columns depict the results of road detection by the KNN, DT and SVM approaches after integration with connected components analysis. Road parts in the main images of Figures 4 and 5 are evidently less or more covered by other occlusions with similar reflectance, making accurate road part extraction from images difficult. This phenomenon is due to the objects with the same spectral features, which possibly become visible as a road section in the extracted image. Consequently, OBIA, connected components analysis, and morphological operations were applied along with segmentation and classification method to obtain additional information, such as texture and geometry, and eliminate irrelevant road components and noises to improve the accuracy. As shown in Figures 4 and 5, the proposed integration of KNN, DT, and SVM methods with connected components could generally extract accurate road section from orthophoto images. However, the three proposed classification methods demonstrated better performance for extracting road from images in Figure 5 with parameters values of scale $=20$, shape $=0.2$ and compactness $=0.6$ than those in Figure 4 with parameters values of scale $=50$, shape $=0.5$ and compactness $=0.3$. In both figures, the proposed SVM method could produce better qualitative results for road extraction with less false positive (FPs) prediction (shown as blue color) than other methods while KNN method predicted more FPs and less false negative (FNs) (shown as yellow color) and generated low-quality visualization results compared to other approaches.
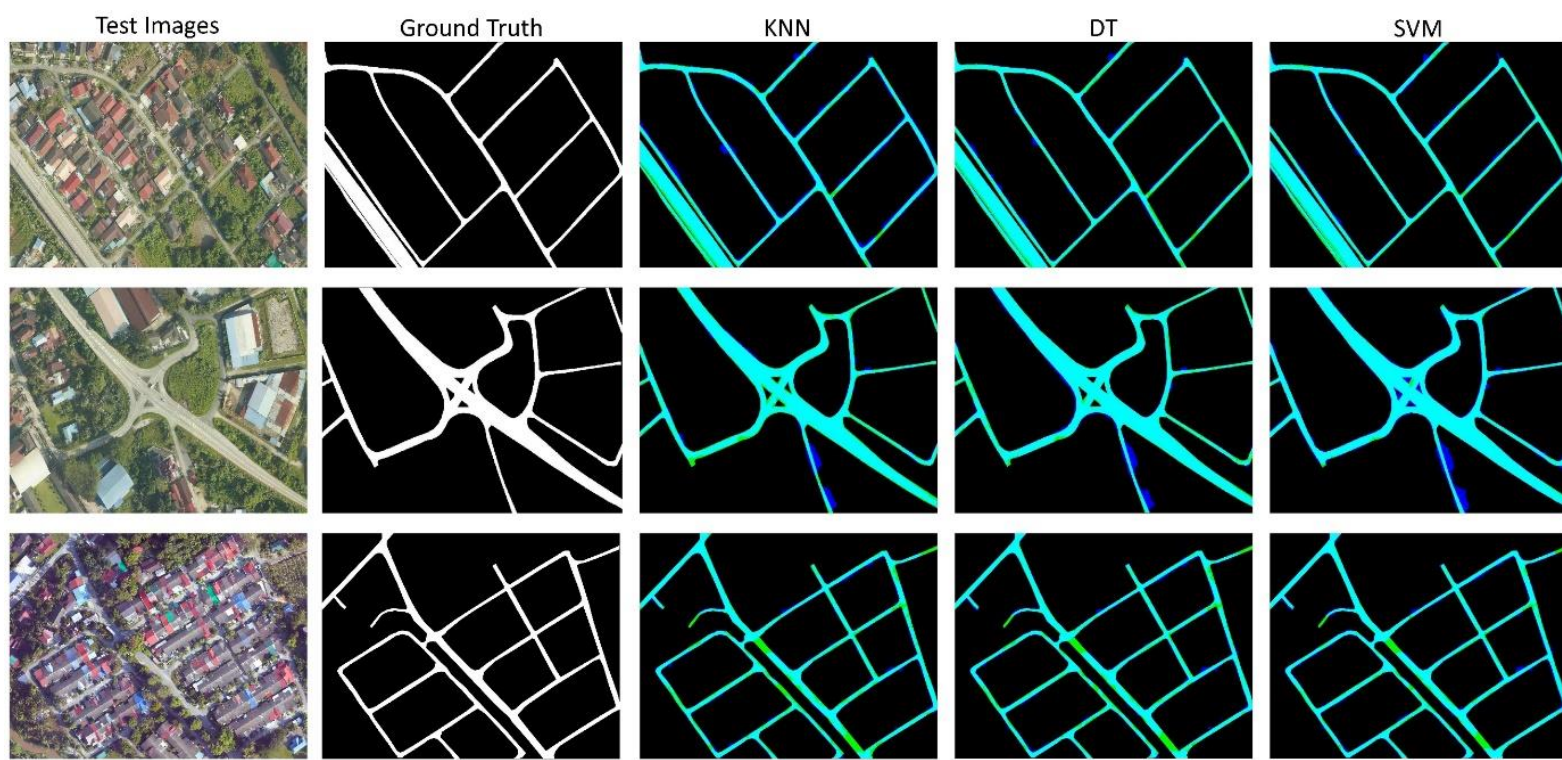

Fig. 4. Extracted road class from orthophoto images with scale $=50$, shape $=0.5$ and compactness $=0.3$. First and second columns show the original image road label, respectively while third, fourth and fifth columns show the results of road detection by KNN, DT and SVM approaches, respectively. 

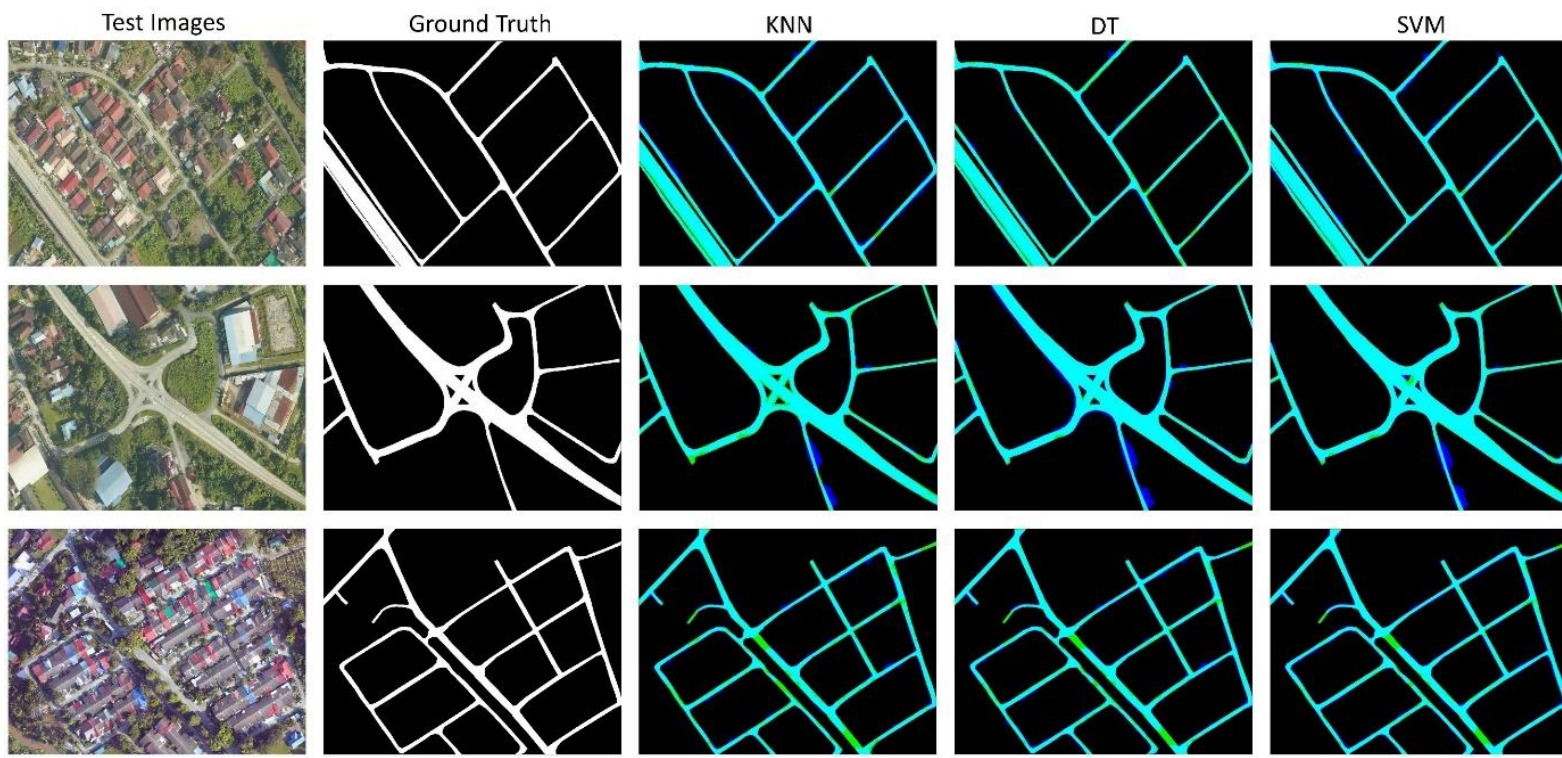

Fig. 5. Extracted road class from orthophoto images with scale $=20$, shape $=0.2$ and compactness $=0.6$. First and second columns show the original image road label, respectively while third, fourth and fifth columns show the results of road detection by KNN, DT and SVM approaches, respectively.

A confusion matrix with four main factors (true negative (TN), false negative (FN), true positive (TP), and false positive (FP)) was used for assessing the accuracy of the suggested approach because road part extraction from remote sensing image is a binary classification.

The amount of incorrectly classified pixels in terms of road section is called FP, while the amount of incorrectly extracted pixels related to non-road part is defined as FN. TP is considered being the amount of accurately classified road pixels, and TN is the accurately classified non-road pixels (Wei, Wang, \& Xu, 2017). Several main metrics, such as recall, F1score, and precision, were considered based on the parameters of the confusion matrix to evaluate the capability of the introduced approach in road network extraction from orthophoto images. Table 1 demonstrates the quantitative results achieved by the proposed methods for Figure 4 and those for Figure 5 are presented in Table 2.

Table 1. Evaluated metrics for different methods (Figure 4). Best values are in bold and second-best values are underlined.

\begin{tabular}{c|c|c|c|c}
\hline & & KNN & DT & SVM \\
\hline \multirow{4}{*}{ Image1 } & Recall & 0.8833 & 0.8305 & 0.8485 \\
\cline { 2 - 5 } & Precision & 0.8112 & 0.8957 & 0.8765 \\
\cline { 2 - 5 } & F1-score & 0.8457 & 0.8619 & 0.8623 \\
\hline \multirow{4}{*}{ Image2 } & Recall & 0.8881 & 0.9025 & 0.9326 \\
\cline { 2 - 5 } & Precision & 0.9095 & 0.9161 & 0.9044 \\
\cline { 2 - 5 } & F1-score & 0.8987 & 0.9092 & 0.9182 \\
\hline \multirow{2}{*}{ Image3 } & Recall & 0.7851 & 0.8058 & 0.8547 \\
\cline { 2 - 5 } & Precision & 0.8998 & 0.8967 & 0.8823 \\
\hline
\end{tabular}




\begin{tabular}{c|c|c|c|c}
\hline & F1-score & 0.8386 & 0.8488 & 0.8683 \\
\hline \multirow{4}{*}{ Average } & Recall & $\underline{0.8522}$ & 0.8463 & $\mathbf{0 . 8 7 8 6}$ \\
\cline { 2 - 5 } & Precision & 0.8735 & $\mathbf{0 . 9 0 2 8}$ & $\underline{0.8877}$ \\
\cline { 2 - 5 } & F1-score & 0.8610 & $\underline{0.8733}$ & $\mathbf{0 . 8 8 2 9}$ \\
\hline
\end{tabular}

Table 2. Evaluated metrics for different methods (Figure 5). Best values are in bold and second-best values are underlined.

\begin{tabular}{c|c|c|c|c}
\hline & & KNN & DT & SVM \\
\hline \multirow{4}{*}{ Image1 } & Recall & 0.8966 & 0.8492 & 0.8922 \\
\cline { 2 - 5 } & Precision & 0.8442 & 0.9167 & 0.8982 \\
\cline { 2 - 5 } & F1-score & 0.8696 & 0.8817 & 0.8952 \\
\hline \multirow{4}{*}{ Image2 } & Recall & 0.8952 & 0.9318 & 0.9218 \\
\cline { 2 - 5 } & Precision & 0.9144 & 0.9023 & 0.9223 \\
\cline { 2 - 5 } & F1-score & 0.9047 & 0.9168 & 0.9220 \\
\hline \multirow{4}{*}{ Image3 } & Recall & 0.8064 & 0.8475 & 0.8809 \\
\cline { 2 - 5 } & Precision & 0.8865 & 0.8722 & 0.8651 \\
\cline { 2 - 5 } & F1-score & 0.8446 & 0.8597 & 0.8730 \\
\hline \multirow{4}{*}{ Average } & Recall & 0.8661 & 0.8762 & $\mathbf{0 . 8 9 8 3}$ \\
\cline { 2 - 5 } & Precision & 0.8817 & $\mathbf{0 . 8 9 7 1}$ & 0.8952 \\
\cline { 2 - 5 } & F1-score & 0.8730 & $\underline{0.8861}$ & $\mathbf{0 . 8 9 6 7}$ \\
\hline
\end{tabular}

\section{Discussion}

Based on Table 1, the average percentage of $\mathrm{F} 1$-score metric is $86.10 \%, 87.33 \%$, and $88.29 \%$ for KNN, DT and SVM methods, respectively. Meanwhile, the percentage of such metric presented in Table 2 is $87.30 \%, 88.61 \%$, and $89.67 \%$ for KNN, DT and SVM, respectively. The suggested approaches evidently showed satisfactory performance in terms of road extraction from orthophoto images. However, the accuracy of specific measurements is slightly higher for all the methods in Figure 5 (with scale $=20$, shape $=0.2$ and compactness $=0.6$ ) than those in Figure 4 (with scale $=50$, shape $=0.5$ and compactness $=0.3$ ). As illustrated in Table 1 and 2 , the precision factor percentage is high for the DT model compared with that of the two other methods. However, the SVM model achieved a higher percentage in recall and F1-score than that of the two other methods, which demonstrates the effectiveness of the model for road extraction. In both tables, the KNN method was ranked the least in road detection. The poor road extraction performance of the KNN technique is related to its prediction of a large number of FPs and a smaller number of FNs, which results in poor accuracy. In contrast, the SVM model was ranked the number-one in road extraction in both. In fact, the SVM model could improve the results of F1-score to $2.19 \%$ and $0.96 \%$ compared to KNN and DT, respectively for Figure 4 and $2.37 \%$ and $1.06 \%$, respectively for Figure 5. Figure 6 illustrates the average accuracy of the metrics 
achieved using the proposed road extraction methods for Figure 4 and 5. The vertical and horizontal axes shows the average percentage of accuracy and the three accuracy assessment metrics, respectively. As displayed in Figure 6, SVM model could achieve better quantitative results than KNN and DT. However, all the three proposed models showed a deficiency in road extraction when road parts are covered by occlusions, such as vehicles, shadows, vegetation, and buildings, and predicted more FP pixels. In addition, we measured the computational time of the proposed methods applied on the three images, which the average running time among the approaches is shown in Table 3. As it is obvious, KNN method takes more time than DT and SVM for training with the average running time of 147.33. The reason is that we have to ascertain the value of parameter $\mathrm{K}$ (number of nearest neighbors) and the type of distance to be utilized. Therefore, the computation time is much as the model requires measuring the distance of every query instance to all training samples.

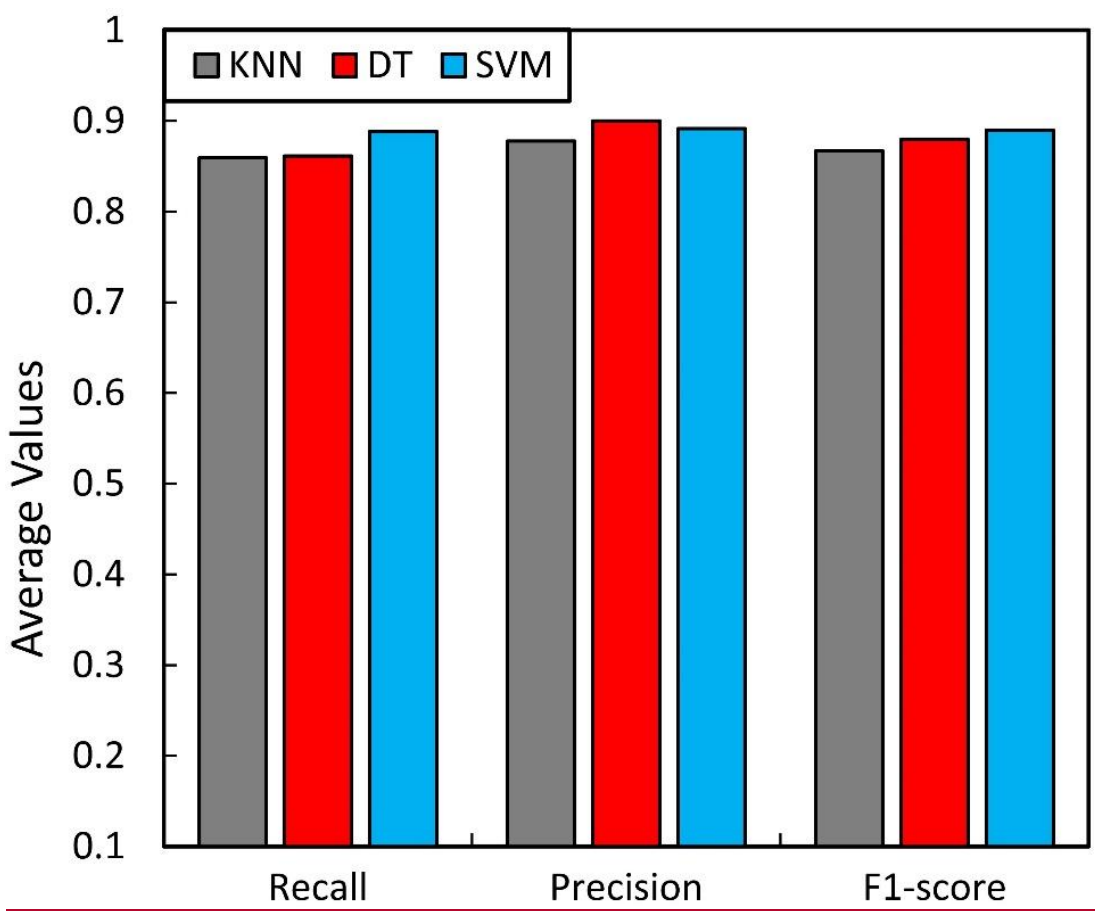

Fig. 6. Comparison of average performance metrics achieved by the proposed methods for road extraction.

Table 3. Computational time comparison of various approaches. Here, the time is measured in second.

\begin{tabular}{c|c|c|c|c}
\hline \multirow{2}{*}{ Methods } & \multicolumn{4}{|c}{ Images } \\
\cline { 2 - 5 } & Image1 & Image2 & Image3 & Average \\
\hline DT & 140 & 104 & 139 & 127.66 \\
\hline KNN & 142 & 105 & 195 & 147.33 \\
\hline SVM & 141 & 106 & 172 & 139.66 \\
\hline
\end{tabular}

In addition, the efficiency of the introduced approaches was compared with that of other works to demonstrate the effectiveness of the model for road extraction from orthophoto imagery. The 
average percentage of recall, precision and F1-score metrics were considered for comparison. A method for road extraction from Ziyuan-3 satellite images based on spectral-spatial classification and shape features was introduced by (Shi, et al., 2014). Recall, precision and F1-score metrics were calculated for the accuracy assessment, in which the average values were obtained caught for comparison. Miao, Wang, Shi, and Zhang (2014) extracted road sections from remotely sensed images according to a fusion method of geodesic, kernel density, and tensor voting techniques. They evaluated recall, precision and F1-score measures to assess the performance, in which the average amount is obtained for comparison with the suggested techniques in this paper. A technique for road extraction from different high-resolution remote sensing images was also introduced by (Maboudi, et al., 2017), in which the average percentage of recall, precision and F1-score factors are obtained for comparison. Table 4 depicts the average amount of performance metrics for the proposed methods in this study and other prior studies.

Table 4. Performance factors of different proposed methods compared with various previous studies. Best values are in bold.

\begin{tabular}{cccc}
\hline Methods & Recall & Precision & F1-score \\
\hline Proposed DT & 0.8762 & 0.8971 & 0.8861 \\
Proposed KNN & 0.8661 & 0.8817 & 0.8730 \\
Proposed SVM & $\mathbf{0 . 8 9 8 3}$ & 0.8952 & $\mathbf{0 . 8 9 6 7}$ \\
Shi et al. (2014) & 0.79 & 0.77 & 0.7798 \\
Miao et al. (2014) & 0.87 & $\mathbf{0 . 9 2}$ & 0.8943 \\
Maboudi et al. (2017) & 0.86 & 0.91 & 0.8842 \\
\hline
\end{tabular}

Table 4 shows that the three proposed SVM method in this study demonstrated a higher percentage in F1-score factor compared with that from previous works. The DT method is ranked third with $88.61 \%$, while SVM is ranked first with $89.67 \%$. By contrast, the average value of F1score for the second-best method (Miao, et al. (2014)) is $89.43 \%$, which could achieve better results than the proposed KNN and DT methods. Miao et al. (2014) also achieved a high precision amount with 92\%, which is more than the average percentage of precision for the three proposed methods with $89.52 \%, 89.71 \%$, and $88.17 \%$ for SVM, DT, and KNN. The decreasing accuracy for the proposed methods is due to the high FP amount prediction, which affected the percentage of precision. Also, Shi et al. (2014) obtained the lowest amount of F1-score with $77.98 \%$, indicating that their method was ineffective in road extraction. By comparing the quantitative 
results, it can be seen that the three proposed classification methods integrated with connected components analysis demonstrated efficiency in road extraction from orthophoto images.

\section{Conclusion}

In the current research, a new integrated model of segmentation and classification methods with connected components analysis is introduced to extract road parts from VHR orthophoto images. The introduced model includes three main steps. First, multiresolution segmentation approach was applied to segment orthophoto images. The obtained results are then processed by the classification methods, such as SVM, KNN, and DT, to categorize the image into road and non-road sections. Training the approaches not only utilized spectral information but also included texture and geometry information to improve the accuracy of the model. Finally, connected components labeling and morphological operations were performed to delete some components that do not belong to the road section, fill the gaps, and enhance the model performance for road extraction. Three different orthophoto images were used for applying the methods, and final outcomes proved that the suggested models were capable of road extraction with satisfactory results. The roads layer was manually digitized to compare the results achieved by the suggested approaches, and three common accuracy metrics, such as recall, precision, and F1-score, were calculated. The average metrics percentage obtained by the suggested methods were $87.62 \%, 89.71 \%$, and $88.61 \%$, respectively, for DT; $86.61 \%, 88.17 \%$, and $87.30 \%$, respectively, for KNN; and $89.83 \%, 89.52 \%$, and $89.67 \%$, respectively, for SVM. The results from different accuracy assessment factors were also compared with those of other previous studies, which showed that the integrated model was still efficient in terms of accurate road region extraction from orthophoto images. The novelty of the proposed integrated method lies in its capability to distinguish and extract straight and curved road parts. However, some parts of the road in the image are entirely covered by trees and shadows, making accurate road extraction from these parts difficult. Therefore, this difficulty is considered a limitation and deficiency of the integrated approach.

Author Contributions: Conceptualization, A.A. and B.P.; methodology and formal analysis, A.A.; data capturing and curation, A.A. and B.P.; writing—original draft preparation, A.A.; writing - review and editing, B.P.; supervision and funding, B.P.

Funding: This research is supported by the Centre for Advanced Modelling and Geospatial Information Systems (CAMGIS) in the University of Technology Sydney (UTS).

Conflict of Interest: The authors declare no conflict of interest. 
Acknowledgments: The authors wish to thank the Department of Mineral and Geosciences, the Department of Surveying Malaysia, the Federal Department of Town and Country Planning Malaysia for the data provided. This research was funded by the Centre for Advanced Modelling and Geospatial Information Systems (CAMGIS), Faculty of Engineering \& IT, University of Technology Sydney.

\section{References}

Aasen, H., Honkavaara, E., Lucieer, A., \& Zarco-Tejada, P. (2018). Quantitative remote sensing at ultra-high resolution with uav spectroscopy: A review of sensor technology, measurement procedures, and data correction workflows. Remote Sensing, 10, 1091.

Abdollahi, A., Bakhtiari, H. R. R., \& Nejad, M. P. (2018). Investigation of SVM and level set interactive methods for road extraction from google earth images. Journal of the Indian Society of Remote Sensing, 46, 423-430.

Abdollahi, A., Pradhan, B., \& Shukla, N. (2019). Extraction of road features from UAV images using a novel level set segmentation approach. International Journal of Urban Sciences, 1-15.

Abdollahi, A., Pradhan, B., Shukla, N., Chakraborty, S., \& Alamri, A. (2020). Deep learning approaches applied to remote sensing datasets for road extraction: a state-of-the-art teview. Remote Sensing, 12(9), 1444.

Ahmad, A., \& Quegan, S. (2012). Analysis of maximum likelihood classification on multispectral data. Applied Mathematical Sciences, 6, 6425-6436.

Akbulut, Y., Sengur, A., Guo, Y., \& Smarandache, F. (2017). NS-k-NN: Neutrosophic set-based knearest neighbors classifier. Symmetry, 9, 179.

Alshehhi, R., \& Marpu, P. R. (2017). Hierarchical graph-based segmentation for extracting road networks from high-resolution satellite images. ISPRS Journal of Photogrammetry and Remote Sensing, 126, 245-260.

Bakhtiari, H. R. R., Abdollahi, A., \& Rezaeian, H. (2017). Semi automatic road extraction from digital images. The Egyptian Journal of Remote Sensing Space Science, 20, 117-123.

Blaschke, T. (2010). Object based image analysis for remote sensing. ISPRS Journal of Photogrammetry and Remote Sensing, 65, 2-16.

Burges, C. J. C. (1998). A tutorial on support vector machines for pattern recognition. Data Mining Knowledge Discovery, 2, 121-167.

Chaudhuri, D., Kushwaha, N., \& Samal, A. (2012). Semi-automated road detection from high resolution satellite images by directional morphological enhancement and segmentation techniques. IEEE Jjournal of Sfelected Ttopics in Atpplied Eearth Ö 1544.

Courtrai, L., \& Lefèvre, S. (2016). Morphological path filtering at the region scale for efficient and robust road network extraction from satellite imagery. Pattern Recognition Letters, 83, 195204.

Da-Ming, Z., Xiang, W., \& Chun-Li, L. (2011). Road extraction based on the algorithms of MRF and hybrid model of SVM and FCM. In Image and Data Fusion (ISIDF), International Symposium on Image and Data Fusion. Tengchong, Yunnan, China, 1-4.

Das, S., Mirnalinee, T., \& Varghese, K. (2011). Use of salient features for the design of a multistage framework to extract roads from high-resolution multispectral satellite images. IEEE Transactions on Geoscience Remote Ssensing, 49, 3906-3931.

Fauvel, M., Chanussot, J., \& Benediktsson, J. A. (2012). A spatial-spectral kernel-based approach for the classification of remote-sensing images. Pattern Recognition Letters, 45, 381-392.

Ferraz, A., Mallet, C., \& Chehata, N. (2016). Large-scale road detection in forested mountainous areas using airborne topographic lidar data. ISPRS Journal of Photogrammetry Remote Sensing, 112, 23-36. 
Gao, X., Sun, X., Zhang, Y., Yan, M., Xu, G., Sun, H., Jiao, J., \& Fu, K. (2018). An end-to-end neural network for road extraction from remote sensing imagery by multiple feature pyramid network. IEEE Access, 6, 39401-39414.

Grinias, I., Panagiotakis, C., \& Tziritas, G. (2016). MRF-based segmentation and unsupervised classification for building and road detection in peri-urban areas of high-resolution satellite images. ISPRS Journal of Photogrammetry Remote Sensing, 122, 145-166.

Grote, A., Heipke, C., \& Rottensteiner, F. (2012). Road network extraction in suburban areas. The Photogrammetric Record, 27, 8-28.

Guo, X., Huang, X., Zhang, L., Zhang, L., Plaza, A., \& Benediktsson, J. A. (2016). Support tensor machines for classification of hyperspectral remote sensing imagery. IEEE Transactions on Geoscience Remote Sensing, 54, 3248-3264.

Huang, K., Li, S., Kang, X., \& Fang, L. (2016). Spectral-spatial hyperspectral image classification based on KNN. Sensing and Imaging, 17, 1.

Huang, X., Lu, Q., \& Zhang, L. (2014). A multi-index learning approach for classification of highresolution remotely sensed images over urban areas. ISPRS Journal of Photogrammetry Remote Sensing, 90, 36-48.

Kamangir, H., Momeni, M., \& Satari, M. (2017). Automatic centerline extraction of covered roads by surrounding objects from high resolution satellite images. ISPRS-International Archives of the Photogrammetry, Remote Sensing Spatial Information Sciences, 111-116.

Khesali, E., Zoej, M. J. V., Mokhtarzade, M., \& Dehghani, M. (2016). Semi automatic road extraction by fusion of high resolution optical and radar images. Journal of the Indian Society of Remote Sensing, 44, 21-29.

Li, J., Jin, L., Fei, S., \& Ma, J. (2014). Robust urban road image segmentation. In Proceeding of the 11th World Congress on Intelligent Control and Automation, Shenyang, China, 2923-2928. https://doi.org/10.1109/WCICA.2014.7053193.

Li, Y., Peng, B., He, L., Fan, K., Li, Z., \& Tong, L. (2019). Road extraction from unmanned aerial vehicle remote sensing images based on improved neural networks. Sensors, 19, 4115.

Liu, B., Wu, H., Wang, Y., \& Liu, W. (2015). Main road extraction from zy-3 grayscale imagery based on directional mathematical morphology and vgi prior knowledge in urban areas. PloS one, 10, $1-16$.

Maboudi, M., Amini, J., Hahn, M., \& Saati, M. (2017). Object-based road extraction from satellite images using ant colony optimization. International Journal of Remote Sensing, 38, 179-198.

Miao, Z., Shi, W., Gamba, P., \& Li, Z. (2015). An object-based method for road network extraction in VHR satellite images. IEEE Journal of Selected Topics in Applied Earth Observations and Remote Sensing, 8, 4853-4862.

Miao, Z., Shi, W., Zhang, H., \& Wang, X. (2012). Road centerline extraction from high-resolution imagery based on shape features and multivariate adaptive regression splines. IEEE Geoscience Remote Sensing Letters, 10, 583-587.

Miao, Z., Wang, B., Shi, W., \& Zhang, H. (2014). A semi-automatic method for road centerline extraction from VHR images. IEEE Geoscience and Remote Sensing Letters, 11, 1856-1860.

Mishra, P., Singh, D., \& Yamaguchi, Y. (2011). Land cover classification of PALSAR images by knowledge based decision tree classifier and supervised classifiers based on SAR observables. Progress In Electromagnetics Research, 30, 47-70.

Mnih, V., \& Hinton, G. E. (2010). Learning to Detect Roads in High-Resolution Aerial Images. In. Berlin, Heidelberg, 210-223. https://doi.org/10.1007/978-3-642-15567-3 16.

ke, T. (2010). Land cover change assessment using decision trees, support vector machines and maximum likelihood classification algorithms. International Journal of Applied Earth Observation Geoinformation, 12, S27-S31.

Qian, Y., Zhou, W., Yan, J., Li, W., \& Han, L. (2015). Comparing machine learning classifiers for object-based land cover classification using very high resolution imagery. Remote Sensing, 7, 153-168.

Ratle, F., Camps-Valls, G., \& Weston, J. (2010). Semisupervised neural networks for efficient hyperspectral image classification. IEEE Transactions on Geoscience Remote Sensing, 48, 2271-2282. 
Revathi, M., \& Sharmila, M. (2013). Automatic road extraction using high resolution satellite images based on level set and mean shift methods. In 2013 Fourth International Conference on Computing, Communications and Networking Technologies (ICCCNT), Tiruchengode, India, 1-7. https://doi.org/10.1109/ICCCNT.2013.6726766.

Rezaee, M., \& Zhang, Y. (2017). Road detection using deep neural network in high spatial resolution images. In 2017 Joint Urban Remote Sensing Event (JURSE), 1-4.

Rodriguez-Galiano, V. F., Ghimire, B., Rogan, J., Chica-Olmo, M., \& Rigol-Sanchez, J. P. (2012). An assessment of the effectiveness of a random forest classifier for land-cover classification. ISPRS Journal of Photogrammetry Remote Sensing, 67, 93-104.

Saba, F., Valadan Zoej, M. J., \& Mokhtarzade, M. (2016). Optimization of Multiresolution Segmentation for Object-Oriented Road Detection from High-Resolution Images. Canadian Journal of Remote Sensing, 42, 75-84.

Sghaier, M. O., \& Lepage, R. (2016). Road extraction from very high resolution remote sensing optical images based on texture analysis and beamlet transform. IEEE Journal of Selected Topics in Applied Earth Observations and Remote Sensing, 9, 1946-1958.

Shen, Y., Ai, T., \& Yang, M. (2019). Extracting centrelines from dual-line roads using superpixel segmentation. IEEE Access, 7, 15967 - 15979.

Shi, W., Miao, Z., Wang, Q., \& Zhang, H. (2014). Spectral-spatial classification and shape features for urban road centerline extraction. IEEE Geoscience Remote Sensing Letters, 11, 788-792.

Singh, P. P., \& Garg, R. (2013). Automatic road extraction from high resolution satellite image using adaptive global thresholding and morphological operations. Journal of the Indian Society of Remote Sensing, 41, 631-640.

Sujatha, C., \& Selvathi, D. (2015). Connected component-based technique for automatic extraction of road centerline in high resolution satellite images. EURASIP Journal on Image Video Processing, 2015, 8 .

Unsalan, C., \& Sirmacek, B. (2012). Road network detection using probabilistic and graph theoretical methods. IEEE Transactions on Geoscience and Remote Sensing, 50, 4441-4453.

Valero, S., Chanussot, J., Benediktsson, J. A., Talbot, H., \& Waske, B. (2010). Advanced directional mathematical morphology for the detection of the road network in very high resolution remote sensing images. Pattern Recognition Letters, 31, 1120-1127.

Vijayan, S. C., \& Jyothy, R. (2016). Histogram Based Connected Component Analysis for Character Segmentation. International Journal of Scientific Research Publications, 6, 200-202.

Wang, M., \& Li, R. (2014). Segmentation of High Spatial Resolution Remote Sensing Imagery Based on Hard-Boundary Constraint and Two-Stage Merging. IEEE Transactions on Geoscience and Remote Sensing, 52, 5712-5725.

Wei, Y., Wang, Z., \& Xu, M. (2017). Road Structure Refined CNN for Road Extraction in Aerial Image. IEEE Geosci. Remote Sensing Letters, 14, 709-713.

Yadav, P., \& Agrawal, S. (2018). Road network identification and extraction in satellite imagery using otsu's method and connected component analysis. In International Archives of the Photogrammetry, Remote Sensing Spatial Information Sciences. Dehradun, India, 91-98.

Zhang, J., Chen, L., Zhuo, L., Geng, W., \& Wang, C. (2018). Multiple saliency features based automatic road extraction from high-resolution multispectral satellite images. Chinese Journal of Electronics, 27, 133-139. 


\section{Highlights}

- Classification methods are presented for image classification into road and non-road

- OBIA is utilized for getting further information

- Trivial opening is applied for road extraction

- Morphological closing is applied for filling holes 
ORCID Information:
Abolfazl Abdollahi: $\underline{\text { https://orcid.org/0000-0002-1704-4670 }}$
Biswajeet Pradhan: $\underline{\text { https://orcid.org/0000-0001-9863-2054 }}$

ORCID Information:
Abolfazl Abdollahi: $\underline{\text { htps://orcid.org/0000-0002-1704-4670 }}$
Biswajeet Pradhan: $\underline{\text { https://orcid.org/0000-0001-9863-2054 }}$

ORCID Information:
Abolfazl Abdollahi: $\underline{\text { htps://orcid.org/0000-0002-1704-4670 }}$
Biswajeet Pradhan: $\underline{\text { https://orcid.org/0000-0001-9863-2054 }}$

ORCID Information

ORCID Information

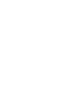

ORCID Information:
Abolfazl Abdollahi: $\underline{\text { htps://orcid.org/0000-0002-1704-4670 }}$
Biswajeet Pradhan: $\underline{\text { https://orcid.org/0000-0001-9863-2054 }}$

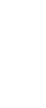

.

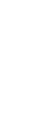

ORCID Information:
Abolfazl Abdollahi: $\underline{\text { htps://orcid.org/0000-0002-1704-4670 }}$
Biswajeet Pradhan: $\underline{\text { https://orcid.org/0000-0001-9863-2054 }}$

ORCID Information:
Abolfazl Abdollahi: $\underline{\text { https://orcid.org/0000-0002-1704-4670 }}$
Biswajeet Pradhan: $\underline{\text { https://orcid.org/0000-0001-9863-2054 }}$

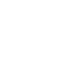






\section{CRediT authorship contribution statement}

Abolfazl Abdollahi: Conceptualization, Methodology, Modelling, Writing original draft. Biswajeet Pradhan: Conceptualisation, Supervision, Data capturing and curation, Validation, Visualization, Review \& Editing, Funding. 
Conflicts of Interest: The authors declare no conflict of interest. 
